\title{
Impact of Early Exposure to Cefuroxime on the Composition of the Gut Microbiota in Infants Following Cesarean Delivery
}

Kamal, Shamrulazhar S.; Hyldig, Nana; Krych, Lukasz; Greisen, Gorm; Krogfelt, Karen A.; Zachariassen, Gitte; Nielsen, Dennis S.

Published in:

Journal of Pediatrics

DOI:

10.1016/j.jpeds.2019.03.001

Publication date:

2019

Document version

Peer reviewed version

Document license:

CC BY-NC-ND

Citation for published version (APA):

Kamal, S. S., Hyldig, N., Krych, L., Greisen, G., Krogfelt, K. A., Zachariassen, G., \& Nielsen, D. S. (2019).

Impact of Early Exposure to Cefuroxime on the Composition of the Gut Microbiota in Infants Following Cesarean Delivery. Journal of Pediatrics, 210, 99-105, e1-e2. https://doi.org/10.1016/j.jpeds.2019.03.001 
Impact of early exposure to Cefuroxime on Composition of Gut Microbiota in infants following Cesarean delivery

Shamrulazhar S. Kamal ${ }^{\mathrm{a}}$, MSc, Nana Hyldig ${ }^{\mathrm{b}}$, PhD, Łukasz Krych ${ }^{\mathrm{a}}$, PhD, Gorm Greisen $^{\mathrm{c}}, \mathrm{MD}, \mathrm{DMSc}$, Karen A Krogfelt ${ }^{\mathrm{d}}, \mathrm{PhD}$, Gitte Zachariassen ${ }^{\mathrm{e}}, \mathrm{MD}, \mathrm{PhD}$, Dennis S. Nielsen ${ }^{\mathrm{a}}$, PhD

${ }^{a}$ Department of Food Science, University of Copenhagen, Denmark; ${ }^{b}$ Department of Gynecology and Obstetrics, Odense University Hospital, Denmark; 'Department of Neonatology, Rigshospitalet, Copenhagen, Denmark; ${ }^{\mathrm{d} D e p a r t m e n t}$ of Bacteria, Parasites and Fungi, Staten Serum Institute, Denmark; ${ }^{e}$ Institute of Clinical Research, Odense University Hospital, Odense, Denmark.

Corresponding author: Dennis S Nielsen, Department of Food Science, University of Copenhagen Rolighedsvej 26, DK-1958, Frederiksberg. Phone +4551330362, E-mail: $\underline{\mathrm{dn} @ \text { food.ku.dk }}$

Short title: Influence of early-life cefuroxime on gut microbiota

Financial disclosure: The authors have no financial relationships relevant to this article to disclose.

Funding source: This study was supported by the Innovation Fund Denmark on funded the Danish Strategic Research Council, NEOMUNE (grant number 12132401) project and the Aase and Ejnar Danielsen Foundation. Shamrulazhar S. 
Kamal is a recipient of a PhD Scholarship Award from Majlis Amanah Rakyat (MARA), Malaysia. The funding sources played no role in study design, collecting, analyzing and interpreting data, writing the manuscript and the decision to publish the study. Authors MSc Kamal and Dr. Nielsen wrote the first draft of the manuscript.

Conflicts of interest: The authors declare no conflicts of interest

Author contributions:

Dr. Zachariassen and Dr. Hyldig conceived and planned the study, organized the intervention and collection of samples and critically revised the manuscript Dr. Krogfelt and DMSc Greisen conceived and planned the study and critically revised the manuscript.

MSc Kamal performed all laboratory analysis, performed bioinformatics and data analysis and drafted the initial manuscript

Dr. Krych performed bioinformatics and data analysis.

Dr. Nielsen conceived and planned the study, did data analysis and drafted the initial manuscript

All authors approved the final manuscript as submitted and agree to be accountable for all aspects of the work 


\begin{abstract}
Objectives: To asses if administrating mothers giving birth by cesarean delivery prophylactic antibiotics either before skin incision or immediately after cutting the umbilical cord influences gut microbiota colonization and antibiotic susceptibility of the gut bacteria in the newborn. Antibiotics administered before skin incision lowers the risk of surgical site infections for the mothers, but also exposes the fetus to the antibiotic.
\end{abstract}

Methods: Forty-two pregnant women scheduled for elective cesarean section were recruited at Odense University Hospital, Denmark, and randomly assigned to receive cefuroxime either before skin incision or immediately after the umbilical cord was cut. Fecal samples were collected from all infants at age 10 days and 9 months. Composition of the gut microbiota was determined by 16S rRNA gene amplicon high-throughput sequencing. Gram-positive cocci and Enterobacteriaceae were isolated and identified before antimicrobial susceptibility tests were performed by disk diffusion.

Results: No clear difference in the composition of the gut microbiota was observed between infants whose mothers received cefuroxime before or after cesarean delivery at neither time point, though surprisingly at 9 months of age, but not at 10 days of age, the number of observed species was higher in infants where mothers received cefuroxime after cord clamping. No differences in antimicrobial susceptibility of Enterobacteriaceae, Enterococcus spp., and Staphylococcus spp. were seen at 10 days. 
Conclusion: Timing of cefuroxime administration to mothers undergoing cesarean section does not have major effects on gut microbiota and bacterial antibiotic resistance traits in infants.

Clinical trials registration: "Antibiotics and Microbiota among newborn Infants", NCT02072798. 


\section{Introduction}

Birth by caesarean delivery is common, with cesarean delivery rates exceeding $50 \%$ in some countries. ${ }^{1}$ In Denmark approximately $20 \%$ of all newborns are born by cesarean delivery. ${ }^{2}$ Women undergoing cesarean delivery compared with vaginal delivery demonstrate an increased risk of postpartum infections with endometritis, urinary tract infection, and wound infection being the most common. ${ }^{3}$ Prophylactic administration of antibiotics to the mother lowers the risk of surgical site infection. It is thus standard cesarean delivery procedure to administer to the mother a single prophylactic dose of cefuroxime $(1500 \mathrm{mg})$ or other broad spectrum antibiotics either shortly before skin incision or immediately after umbilical cord clamping. ${ }^{4}$ While several studies have shown that administering the antibiotics to the mother $30-60$ minutes before skin incision is optimal for lowering SSI risk, ${ }^{5-9}$ this also exposes the fetus to the antibiotic via placental transfer. ${ }^{10}$ To avoid unintended fetal exposure to antibiotics during CS, antibiotics at many hospitals are administered to the mother immediately after the cord is clamped. ${ }^{11}$

At birth, the neonate's gastrointestinal tract is rapidly colonized by microorganisms. ${ }^{12}$ Initially the microbial community composition is relatively simple. ${ }^{13}$ During the first year of life the gut microbiota, influenced by environmental factors (breast vs. formula feeding; introduction of weaning food, exposure to antibiotics etc.), gradually develops into a more adult-like, complex microbiota. ${ }^{14-17}$ Early life gut colonization plays pivotal roles in development of intestinal functions and postnatal immune maturation, which might influence long-term health and risk of development of disorders, e.g. autoimmune diseases. ${ }^{18-21}$ 
High exposure to antibiotics during early life has been shown to influence long-term structure of the gut microbiota and might be a risk factor for the development of certain diseases, such as asthma and obesity. ${ }^{21-26}$ Furthermore, since early life antibiotic exposure increases the occurrence of antibiotic resistant gut bacteria, especially Enterobacteriaceae, which can have serious health implications ${ }^{27}$ it is highly recommended to limit exposure to antibiotics in infants as much as possible. However, short and long-term effect on the developing gut microbiome of very early and brief (i.e. at or around birth) exposure to antibiotics is unclear.

Cefuroxime administered intravenously to the mother shortly before cesarean delivery is cleared from infants' blood plasma approximately three times more slowly than in adults, but nevertheless a single maternal dose of $1500 \mathrm{mg}$ cefuroxime is cleared from the infant within 24 hours. ${ }^{10}$ In the present study we aim to investigate whether maternal exposure to antibiotics shortly before or immediately after parturition influences the gut microbiota of the infant short- and moderately long-term, and whether use of antibiotics in this window of time increases the prevalence of antibiotic resistant gut bacteria.

\section{Methods}

\section{Study Subjects}

This study was performed at the Department of Gynaecology and Obstetrics, Odense University Hospital, Denmark from February 2014 to July 2014 and is registered in Clinical Trials as "Antibiotics and Microbiota among newborn Infants", NCT02072798. Inclusion criteria were planned cesarean delivery, age above 18 years, 
a pre-gestational body mass index (BMI) less than 30. Forty-two women were randomly assigned to receive a single dose of intravenous cefuroxime (1500 mg), either administered 15 to 60 minutes prior to surgical incision $($ Pre, $n=22$ ) or immediately after umbilical cord clamping (Post, $\mathrm{n}=20$ ). Details on caesarean delivery procedure, recruitment procedure and medical ethics have been reported elsewhere. ${ }^{10}$ A restricted block randomization sequence was created with a 1:1 allocation using a fixed block size of 4 . The block size was unknown to the investigators, study nurse and participants during the trial. No stratification was used. A data manager with no clinical involvement in the trial prepared the randomization sequence using computer-generated random numbers. Infants whose mother received cefuroxime before skin excision (Pre) had blood sampled to determine cefuroxime clearance from the blood. ${ }^{10}$ It was deemed unethical to also sample blood from infants not exposed to the antibiotic (Post) and the study consequently not blinded. Study infants were healthy, did not receive any antibiotics postnatally, and were discharged between two to five days after birth. Infant health conditions after hospital discharge were monitored by a trained nurse and early feeding practices (exclusive breast feeding, mix breast feeding and infant formula or exclusive infant formula) were recorded.

During the first 10 days of life 15 infants were exclusively breast fed, 4 were exclusively fed formula milk and 25 infants had received both formula and breast feeding. No diet information was recorded at age of 9 months. Fecal samples were collected from the infants during the nurse/midwife's visit 10 days after birth and 9 months after birth. Fecal samples were obtained from 44 infants at day 10 and from 
42 infants at 9 months (one set of twins withdrew from the study). The fecal samples were transferred into sterile tubes and stored at $-60^{\circ} \mathrm{C}$ or colder until further analyses.

\section{Ethics}

The trial was approved by the Danish Health and Medicines Authority (EudraCT 2012-002068-29), the Committee on Biomedical Research Ethics (S-20130117), Danish Data Protection Agency (2008-58-0035) and was monitored by the local Good Clinical Research Unit (NCT02072798). All mothers gave written consent prior to cesarean delivery and prior to collecting any samples.

\section{DNA extraction}

Approximately $200 \mathrm{mg}$ of fecal sample was weighted into a PowerSoil Bead Tube and total DNA extracted using the MoBio PowerSoil DNA isolation kit (MOBIO Laboratories, Carlsbad, CA, USA), following manufacturer's instructions, except that an initial bead beating step was included to increase cell lysis. Prior to DNA extraction, samples were placed into the PowerBead tubes and heat treated at $65^{\circ} \mathrm{C}$ for $10 \mathrm{~min}$ and then at $95^{\circ} \mathrm{C}$ for $10 \mathrm{~min}$. Subsequently, solution $\mathrm{C} 1$ was added and beadbeating performed in a FastPrep (MP Biomedicals, Santa Ana, CA, USA) using 3 cycles of $15 \mathrm{~s}$ each, at a speed of $6.5 \mathrm{~m} \mathrm{~s}^{-1}$. The DNA concentration was measured using Nanodrop (ND-100, NanoDrop Technologies, USA). Extracted DNA was stored at $-60^{\circ} \mathrm{C}$ until further analysis.

\section{S rRNA gene amplicon sequencing}

The fecal composition of the bacterial microbiota was determined using tag-encoded 16S rRNA gene MiSeq-based (Illumina, CA, USA) high throughput sequencing. The 
V3-V4 region of the 16S rRNA gene was amplified using primers compatible with the Nextera Index Kit (Illumina, CA, USA; adapters in bold): NXt_341_F: 5'TCGTCGgCA GCGTCAgAtg TgtataAgag ACAGCCTAYG GGRBGCASCAG-3' and NXt_806_R: 5'-GTCTCGTGGG CTCGGAGATG TGTAAAGAGA CAGGGACTAC NNGGGTATC TAAT-3'; Library construction and sequencing was carried out as previously described. ${ }^{28}$

\section{Amplicon sequencing data analysis}

The raw dataset containing pair-ended reads was merged, trimmed, filtered from chimeric reads with the USEARCH pipeline, ${ }^{29}$ and subjected to zero radius Operational Taxonomic Units (zOTU) construction using UNOISE. ${ }^{30}$ Taxonomy classification was conducted using the SINTAX algorithm ${ }^{31}$ based on the Ribosomal Database Project (RDP, release 11, update 5, 2016). ${ }^{32}$ The Quantitative Insight Into Microbial Ecology (QIIME) open source software package (version 1.8.0) was used for subsequent analysis steps. 32,33

Weighted, unweighted and generalized UniFrac distance metrics were calculated based on subsampled OTU-tables (14,000 reads per sample) and projected with Principal Coordinate Analysis (PCoA) plots. Permutational Multivariate Analysis of Variance (PERMANOVA) ('Adonis' $\mathrm{R}$ package) was used to evaluate group differences based on weighted, unweighted and generalized UniFrac distance matrices. The differences in taxa abundance and prevalence between categories were estimated with a statistic framework: analysis of composition (ANCOM) of microbes based on non-normalized OTU-table summarized to the species level (all p-values False Discovery Rate (FDR) corrected). ${ }^{33}$ 
Alpha diversity expressed as observed species and Shannon indexes were computed for rarefied OTU tables (14,000 reads per sample) using the alpha rarefaction workflow. Alpha diversity differences were tested by t-test using the non-parametric (Monte Carlo) method (999 permutations) that is implemented in the compare alpha diversity workflow.

The full amplicon sequencing data set including metadata has been uploaded to the European Nucleotide Archive (ENA) under the accession number PRJEB25411.

\section{Bacterial isolation and identification}

The isolation and identification of bacteria from the fecal samples was carried out as described previously. ${ }^{13}$ Briefly, fecal samples from day $10(\sim 200 \mathrm{mg})$ were emulsified and transferred in sterile saline $(1 \mathrm{~mL})$ and mixed thoroughly before being inoculation onto nonselective and selective agar (SSI Diagnostica, Copenhagen, Denmark). Samples were streaked onto Blood agar plates (5\% horse blood), blue agar plates (modified Drigalski) and chocolate agar plates and incubated aerobically at $37^{\circ} \mathrm{C}$ for 24 hours (48 hours for chocolate agar plates). Colonies were selected based on morphology and sub-cultured to get pure isolates. Bacterial isolates were then identified by matrix-assisted laser desorption ionization-time of flight (MALDITOF). ${ }^{34}$ Identified isolates were stored in Luria-Bertani (LB) broth containing 20\% glycerol at $-80^{\circ} \mathrm{C}$.

\section{Antimicrobial Susceptibility testing}

The antibiotic susceptibility of the fecal bacterial isolates was determined by KirbyBauer disk diffusion method according to Clinical Laboratory Standards Institute 
recommendation $^{35}$ using disks containing ampicillin $(10 \mu \mathrm{g})$, ciprofloxacin $(5 \mu \mathrm{g})$, cefuroxime $(30 \mu \mathrm{g})$, gentamicin $(10 \mu \mathrm{g})$ or piperacillin/tazobactam $(100 \mu \mathrm{g} / 10 \mu \mathrm{g})$. The inhibition zone diameter was measured after anaerobic incubation at $37^{\circ} \mathrm{C}$ within 18 to 24 hours and results were categorized as resistant (R), intermediate (I) or susceptible (S) also according to Clinical Laboratory Standards Institute. ${ }^{35}$ 


\section{Results}

\section{Composition of the gut microbiota}

A total of 86 fecal samples were obtained from 44 babies who donated fecal samples at 10 days (Pre, $n=24$; Post $n=20)$ and 9 months of age (Pre, $n=22$; Post, $n=20)$ with two twin set of babies participating at 10 days and only one set of twin babies at 9 months (Figure 1).

High throughput 16S rRNA gene amplicon sequencing was used to determine composition of the gut microbiota. No differences in alpha-diversity measures between the groups were observed at day 10 as determined by Observed Species (Pre $=148 \pm 49$, Post $=151 \pm 59, \mathrm{p}=0.76$; Figure 2, Online) and Shannon Diversity indices (Pre: $3.7 \pm 1$, Post: $4.0 \pm 1, \mathrm{p}=0.60$; not shown). At 9 months of age, the number of Observed Species was elevated in the Post group $($ Pre $=361 \pm 141$, Post $=496 \pm 151$, $\mathrm{p}=0.012$; Figure 2, Online) while the Shannon Diversity Index did not differ significantly between the groups (Pre $=5.2 \pm 1.1$, Post $=6.0 \pm 1.3, \mathrm{p}=0.062$ ).

Unweighted, weighted and generalized UniFrac distance metrics showed that very early life antibiotic exposure via the umbilical cord does not influence gut microbiota composition neither short (10 days) nor longer (9 months) term (Figure 3). When analyzing across the entire cohort diet had no significant effect on gut microbiota composition after 10 days (Figure 4, Online). It should be noted that only 4 of the 44 investigated infants were exclusively formula fed with another 25 infants being both formula and breast fed, and 15 infants being exclusively breastfed at day 10 . 
At the compositional level no taxa were found to differ significantly between Pre and Post groups either at 10 days or 9 months of age (ANCOM, $\mathrm{q}>0.05$ in all cases). At day 10 the gut microbiota composition of both groups were dominated by OTUs belonging to family Enterobacteriaceae and Veillonella dispar, while also Bifidobacterium spp. and Clostridium spp. were present in noticeable numbers (Figure 5). At 9 months, the gut microbiota of the infants was dominated by obligate anaerobes, mainly Faecalibacterium prausnitzii, Blautia and other Clostridales, Lachnospiraceae, Bifidobacterium breve, Bacteriodes and Akkermansia muciniphila (Figure 5).

A total of 153 facultative anaerobic and aerobic bacteria were isolated from fecal samples collected at 10 days of age. Most isolates were Enterococcus spp., (mainly Enterococcus faecalis), Staphylococcus spp. (Staphylococcus epidermidis and S. aureus) and Enterobacteriaceae (mainly Enterobacter cloacae and Klebsiella pneumoniae) (Table 1, Online). The antibiotic susceptibility of the gram-positive isolates was tested against ciprofloxacin, ampicillin and gentamicin (Table 2, Online). No clear differences in antibiotic susceptibility was observed between isolates obtained from Pre and Post group infants, with only 1 (Pre) and 2 (Post) grampositive isolates being resistant to any one of three tested antibiotics. Gram-negative isolates were tested against cefuroxime, ciprofloxacin, ampicillin, gentamicin and pipercillin-tazobactam. Only resistance against ampicillin was widespread among gram-negative isolates from both the Pre and Post group isolates (Table 3) with no differences observed between groups.

\section{Discussion}


When undergoing cesarean section, the mother is administered prophylactic antibiotics to prevent post-surgery complications such as surgical site infection, endometritis and urinary tract infection. ${ }^{3}$ According to the 2012 Danish National Obstetric Guideline on antibiotics and C-sections ${ }^{4}$ women giving birth by $\mathrm{C}$-section are given a single prophylactic intravenous dose $(1500 \mathrm{mg})$ of cefuroxime 15-60 minutes before skin incision. However, hospital practices still differ. At the time of this intervention, the usual practice at Odense University Hospital was to administer a single-dose of cefuroxime immediately after umbilical cord clamping to avoid placental transfer of the antibiotic to the fetus. ${ }^{10}$ Several studies found that administration of prophylactic antibiotics pre-incision reduce maternal wound infections. ${ }^{7-9}$ Lowering the risk wound infection of the mother is important not only for the mother, but also for the new-born - and from a societal perspective it may also help in reducing the cost of extended stays in the hospital.

We have previously showed that cefuroxime does pass the placental barrier, and that a dose of $1500 \mathrm{mg}$ administered to the mother before skin incision is cleared from the blood of the new-born within $24 \mathrm{~h}^{10}$, but whether this very early exposure to antibiotics nevertheless influences GM composition and antibiotic susceptibility of the gut bacteria in the new-born has previously not been investigated. To assess the effect of maternal antibiotic exposure on the infant we investigated gut microbiota composition 10 days and 9 months after birth. Ten days was chosen, as an early time point, where the GM had started to settle after the very dynamic phase immediately birth, when the gut is first colonized. Nine months was chosen as a more long-term time point representing the period after introduction of weaning food and the gradual transition towards a more adult-like gut microbiota. ${ }^{16}$ 
At day 10 the gut microbiota composition of both groups were dominated by to the family Enterobacteriaceae and genus Veillonella, while Bifidobacterium spp. and Clostridium spp. were present in noticeable numbers, too (Figure 5). The dominance of Enterobacteriaceae early in life is in agreement with previous findings, ${ }^{16}$ although the relative abundance of especially Bifidobacterium spp. and Bacteroides tends to be higher in infants born vaginally ${ }^{16}$ compared with the infants born by cesarean delivery in the present study. At 9 months, the gut microbiota of the infants was dominated by obligate anaerobes, mainly Faecalibacterium and Blautia and other Clostridales, Lachnospiraceae, Bifidobacterium, Bacteriodes and Akkermansia which is also in agreement with previous investigations of infant gut microbiota around one year of age (Figure 5). ${ }^{16}$

In terms of Observed Species and Shannon Diversity index, we did not observe any differences between Pre and Post parturition antibiotic administration at 10 days of age, but at 9 months of age we observed a higher number of Observed Species in the Post group (Fig. 1, Online). Neither unweighted, weighted nor generalized Unifrac distance metrics indicated GM compositional differences between Pre and Post groups at 10 days and 9 months of age (Fig. 2). It is important to note that in the present study we investigated only the effect of very early-life exposure to cefuroxime on gut microbiota composition. Maternal exposure to cefuroxime around the time of birth could also influence the maternal milk microbiome.

The elevated number of observed species in the Post group at 9 months of age could indicate an altered succession of bacteria among infants exposed to the antibiotic, but 
this effect would be expected to be more pronounced at 10 days of age. As no other indices indicate an effect of very early-life exposure to cefuroxime on the gut microbiota it seems more likely that the observed difference in observed species between Pre and Post groups at 9 months of age is a result of the substantial reorganization that infant gut microbiota undergoes in the period after weaning ${ }^{16,36}$, but potential long-term effects of very early-life exposure to antibiotics cannot be completely excluded.

Early life antibiotic exposure has previously been reported to increase the frequency of antibiotic resistant bacterial gut microbiota members, especially Enterobacteriaceae. ${ }^{27}$ However, in the present study we observed no differences in antibiotic susceptibility profiles of gram-positive cocci and Enterobacteriaceae isolated from Pre and Post fecal samples (10 days). The observation that a relatively high fraction of the investigated Klebsiella pneumoniae and Enterobacter cloacae isolates were resistant to ampicillin is not surprising, as ampicillin has been widely used to treat bacterial infections for decades.

\section{Acknowledgements}

We would like to thank the clinical staff at the Department of Gynecology and Obstetrics, Odense University Hospital for collecting the fecal samples, and laboratory staff Michala T. Sørensen, for her technical assistant in microbiology analysis at, Statens Serum Institute, Denmark.

\section{References}


[1] Nakamura-Pereira M, do Carmo Leal M, Esteves-Pereira AP, Domingues RM, Torres JA, Dias MA, et al. Use of Robson classification to assess cesarean section rate in Brazil: the role of source of payment for childbirth. Reprod Health. 2016;13:128.

[2] Anon. Obstetriske og føtalmedicinske koder - DSOG. wwwdsogdk/koder-ogkvalitetssikring/obstetriske-og-fotalmedicinske-koder/. 2014 (Online).

[3] Leth RA, Moller JK, Thomsen RW, Uldbjerg N, Norgaard M. Risk of selected postpartum infections after cesarean section compared with vaginal birth: a five-year cohort study of 32,468 women. Acta Obstet Gynecol Scand 2009;88:976-83.

[4] Daugaard AE, A.; Hansen, K.B.; Hein, M.; Helmig, R.B.; Hornshoj, V.G.; Houman, I.; Madsen, L.D.; Petersen, K.B.; Poulsen, V.T.; Rom, L.H. Antibiotika. http://gynobsguidelinedk/sandbjerg/120425\%20ANTIBIOTIKA\%20endelig\%2025\% 204\%2012pdf. 2012 (Online).

[5] Heesen M, Klöhr S, Rossaint R, Allegeaert K, Deprest J, Van de Velde M, et al. Concerning the timing of antibiotic administration in women undergoing caesarean section: a systematic review and meta-analysis. BMJ Open 2013;3:e02028.

[6] Lamont RF, Sobel JD, Kusanovic JP, Vaisbuch E, Mazaki-Tovi S, Kim SK, et al. Current debate on the use of antibiotic prophylaxis for caesarean section. BJOG 2011;118:193-201.

[7] Costantine MM, Rahman M, Ghulmiyah L, Byers BD, Longo M, Wen T, et al. Timing of perioperative antibiotics for cesarean delivery: a metaanalysis. Am J Obstet Gynecol 2008;199:301.e1-6.

[8] Tita AT, Rouse DJ, Blackwell S, Saade GR, Spong CY, Andrews WW. Emerging concepts in antibiotic prophylaxis for cesarean delivery: a systematic review. Obstetrics Gynecol 2009; 113:675-82. 
[9] Baaqeel H, Baaqeel R. Timing of administration of prophylactic antibiotics for caesarean section: a systematic review and meta-analysis. BJOG 2013;120:661-9.

[10] Zachariassen G, Hyldig N, Joergensen JS, Nielsen DS, Greisen G. The half-life and exposure of cefuroxime varied in newborn infants after a Caesarean section. Acta Paed 2016;105:1074-8.

[11] Steinberg JP, Braun BI, Hellinger WC, Kusek L, Bozikis MR, Bush AJ, et al. Timing of antimicrobial prophylaxis and the risk of surgical site infections: results from the Trial to Reduce Antimicrobial Prophylaxis Errors. Ann Surg 2009;250:10-6. [12] Bourlioux P, Koletzko B, Guarner F, Braesco V. The intestine and its microflora are partners for the protection of the host: report on the Danone Symposium "The Intelligent Intestine," held in Paris, June 14, 2002. Am J Clin Nutr 2003;78:675-83.

[13] Smith B, Bode S, Skov TH, Mirsepasi H, Greisen G, Krogfelt KA. Investigation of the early intestinal microflora in premature infants with/without necrotizing enterocolitis using two different methods. Pediatr Res 2012;71:115-20.

[14] Westerbeek EA, van den Berg A, Lafeber HN, Knol J, Fetter WP, van Elburg RM. The intestinal bacterial colonisation in preterm infants: a review of the literature. Clin Nutr 2006;25:361-8.

[15] Dominguez-Bello MG, Costello EK, Contreras M, Magris M, Hidalgo G, Fierer $\mathrm{N}$, et al. Delivery mode shapes the acquisition and structure of the initial microbiota across multiple body habitats in newborns. Proc Nat Acad Sci 2010;107:11971-5.

[16] Backhed F, Roswall J, Peng Y, Feng Q, Jia H, Kovatcheva-Datchary P, et al. Dynamics and stabilization of the human gut microbiome during the first year of life. Cell Host Microbe. 2015;17:690-703.

[17] Mulder IE, Schmidt B, Lewis M, Delday M, Stokes CR, Bailey M, et al. Restricting microbial exposure in early life negates the immune benefits associated 
with gut colonization in environments of high microbial diversity. PLoS ONE. 2011;6:e28279.

[18] Cox LM, Yamanishi S, Sohn J, Alekseyenko AV, Leung JM, Cho I, et al. Altering the intestinal microbiota during a critical developmental window has lasting metabolic consequences. Cell. 2014;158:705-21.

[19] Vallès Y, Artacho A, Pascual-García A, Ferrús ML, Gosalbes MJ, Abellán JJ, et al. Microbial succession in the gut: Directional trends of taxonomic and functional change in a birth cohort of Spanish infants. PLOS Genetics 2014;10:e1004406.

[20] Bisgaard H, Li N, Bonnelykke K, Chawes BL, Skov T, Paludan-Muller G, et al. Reduced diversity of the intestinal microbiota during infancy is associated with increased risk of allergic disease at school age. J Allergy Clin Immunol 2011;128:646-52.

[21] Tanaka S, Kobayashi T, Songjinda P, Tateyama A, Tsubouchi M, Kiyohara C, et al. Influence of antibiotic exposure in the early postnatal period on the development of intestinal microbiota. FEMS Immunol Med Microbiol 2009;56:80-7.

[22] Fouhy F, Ross RP, Fitzgerald GF, Stanton C, Cotter PD. Composition of the early intestinal microbiota: knowledge, knowledge gaps and the use of highthroughput sequencing to address these gaps. Gut Microbes 2012;3:203-20.

[23] Korpela K, Salonen A, Virta LJ, Kekkonen RA, Forslund K, Bork P, et al. Intestinal microbiome is related to lifetime antibiotic use in Finnish pre-school children. Nature Comm 2016;7:10410.

[24] Saari A, Virta LJ, Sankilampi U, Dunkel L, Saxen H. Antibiotic exposure in infancy and risk of being overweight in the first 24 months of life. Pediatrics 2015;135:617-26. 
[25] Azad MB, Bridgman SL, Becker AB, Kozyrskyj AL. Infant antibiotic exposure and the development of childhood overweight and central adiposity. Int $\mathrm{j}$ Obesity 2014;38:1290-8.

[26] Arrieta MC, Stiemsma LT, Dimitriu PA, Thorson L, Russell S, Yurist-Doutsch $\mathrm{S}$, et al. Early infancy microbial and metabolic alterations affect risk of childhood asthma. Sci Trans Med. 2015;7:307ra152-307ra152.

[27] Segal Z, Cohen MJ, Engelhard D, Tenenbaum A, Simckes AM, Benenson S, et al. Infants under two months of age with urinary tract infections are showing increasing resistance to empirical and oral antibiotics. Acta Paed 2016;105:e156-60. [28] Pyndt Jørgensen B, Hansen JT, Krych L, Larsen C, Klein AB, Nielsen DS, et al. A possible link between food and mood: Dietary impact on gut microbiota and behavior in BALB/c mice. PLOS ONE. 2014;9:e103398.

[29] Edgar RC. Search and clustering orders of magnitude faster than BLAST. Bioinformatics 2010;26:2460-1.

[30] Edgar RC. UNOISE2: improved error-correction for Illumina $16 \mathrm{~S}$ and ITS amplicon sequencing. bioRxiv 2016:081257.

[31] Edgar R. SINTAX: a simple non-Bayesian taxonomy classifier for 16S and ITS sequences. bioRxiv 2016:074161.

[32] Cole JR, Wang Q, Fish JA, Chai B, McGarrell DM, Sun Y, et al. Ribosomal Database Project: data and tools for high throughput rRNA analysis. Nucleic Acids Res 2014;42:D633-42.

[33] Mandal S, Van Treuren W, White RA, Eggesbo M, Knight R, Peddada SD. Analysis of composition of microbiomes: a novel method for studying microbial composition. Microb Ecol Health Dis 2015;26:27663. 
[34] Carbonnelle E, Mesquita C, Bille E, Day N, Dauphin B, Beretti JL, et al. MALDI-TOF mass spectrometry tools for bacterial identification in clinical microbiology laboratory. Clin. Biochem 2011;44:104-9.

[35] Patel JB, Clinical, Institute LS. Performance Standards for Antimicrobial Susceptibility Testing: Twenty-forth Informational Supplement: Clinical And Laboratory Standards Institute 2014;M100-S24.

[36] Chu DM, Ma J, Prince AL, Antony KM, Seferovic MD, Aagaard KM. Maturation of the infant microbiome community structure and function across multiple body sites and in relation to mode of delivery. Nat Med 2017;23:314-26. 


\section{Figure Legends}

Figure 1. Study design.

Figure 2 (Online). Rarefaction curves based on Observed Species index (16S rRNA gene amplicon sequencing based). Significant $(\mathrm{p}<0.05)$ differences indicated by *.

Figure 3. Gut microbiota compositional similarities between the Pre and Post groups depicted as Principle Coordinate Analysis (PCoA) plots based on unweighted (u), weighted (w) and generalized (g) UniFrac distance metrics showing no clear differences between the gut microbiota of infants born by caesarean section whose mothers received cefuroxime before (red, Pre) or after (blue, Post) skin incision at 10 days and 9 months of age. PERMANOVA values shown each figure. Gut microbiota composition determined by $16 \mathrm{~S}$ rRNA gene amplicon sequencing.

Figure 4 (Online). The influence of diet (BF, exclusive breast feeding; MME, formula feeding, Both, mixed breast and formula feeding) on gut microbial compositional similarities depicted as PCoA plots based on unweighted $(\mathrm{u})$, weighted $(\mathrm{w})$ and generalized $(\mathrm{g})$ UniFrac distance metrics at 10 days. PERMANOVA results are given in each figure. GM composition determined by $16 \mathrm{~S}$ rRNA gene amplicon sequencing.

\section{Figure 5. The relative distribution of the most abundant taxa}

Relative distribution of the top 20 most abundant taxa at (A) 10 days and (B) 9 months of age determined using 16S rRNA gene amplicon sequencing. 


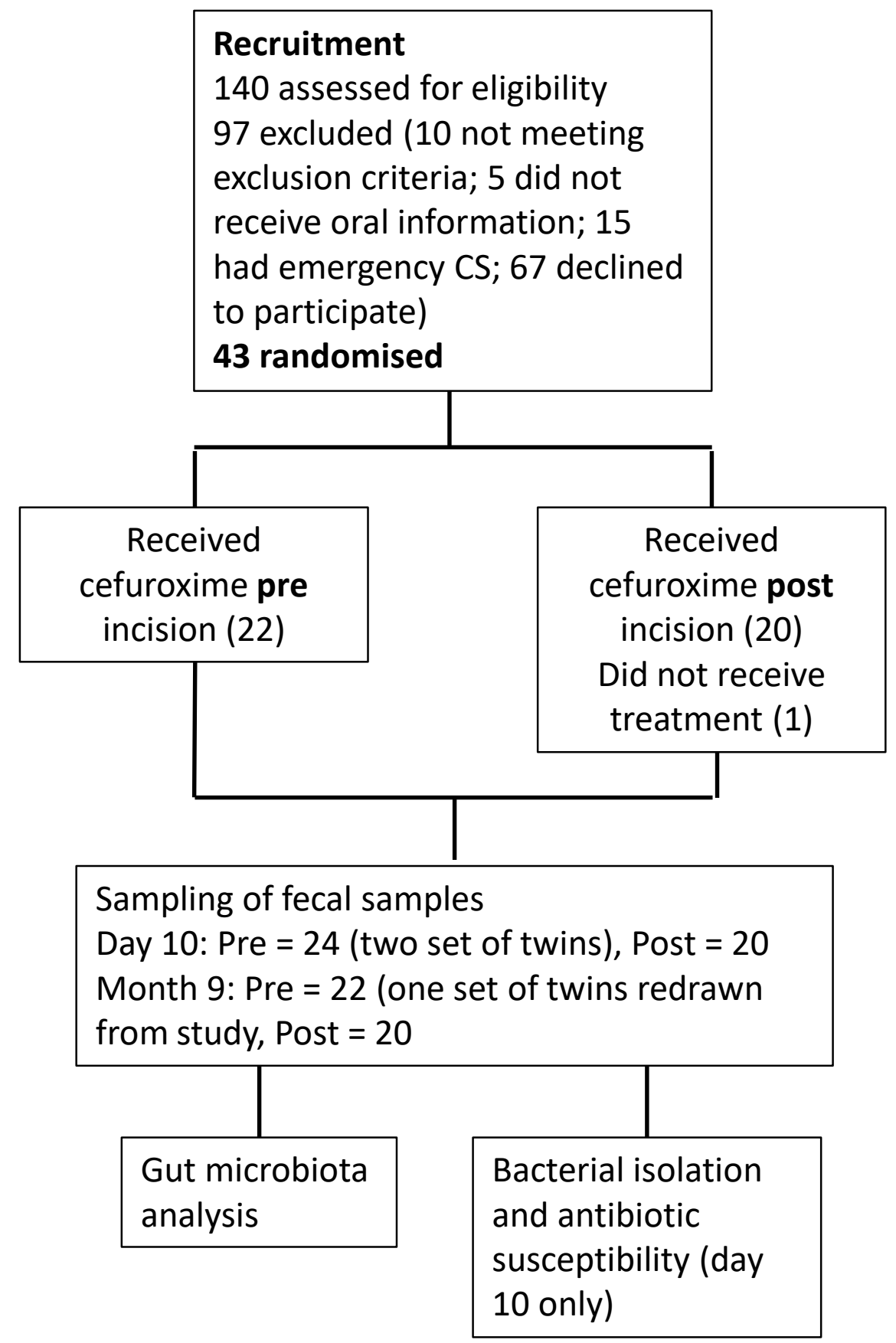

Fig. 1 
Figure 2 (Online)

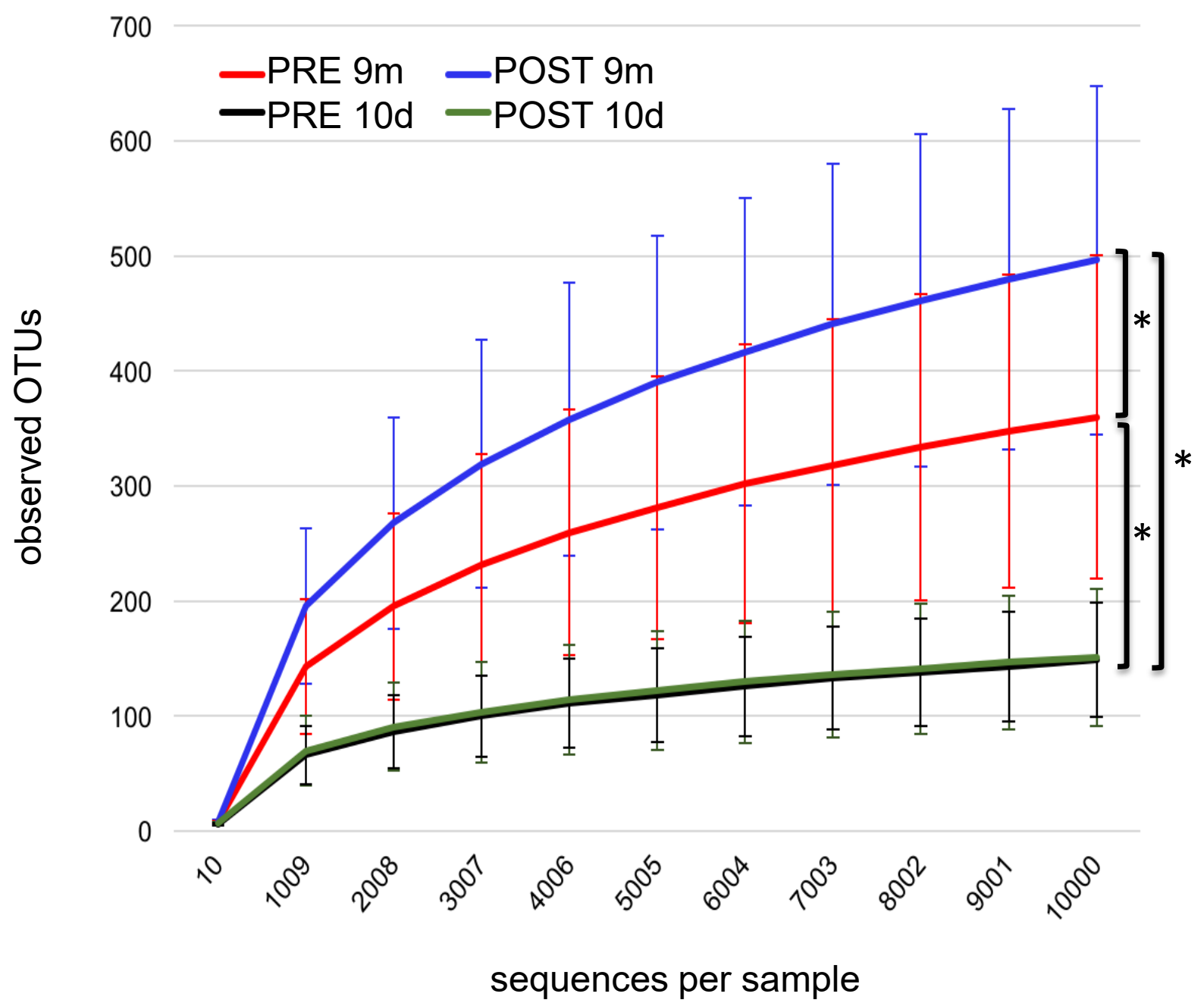




\section{Figure 3}
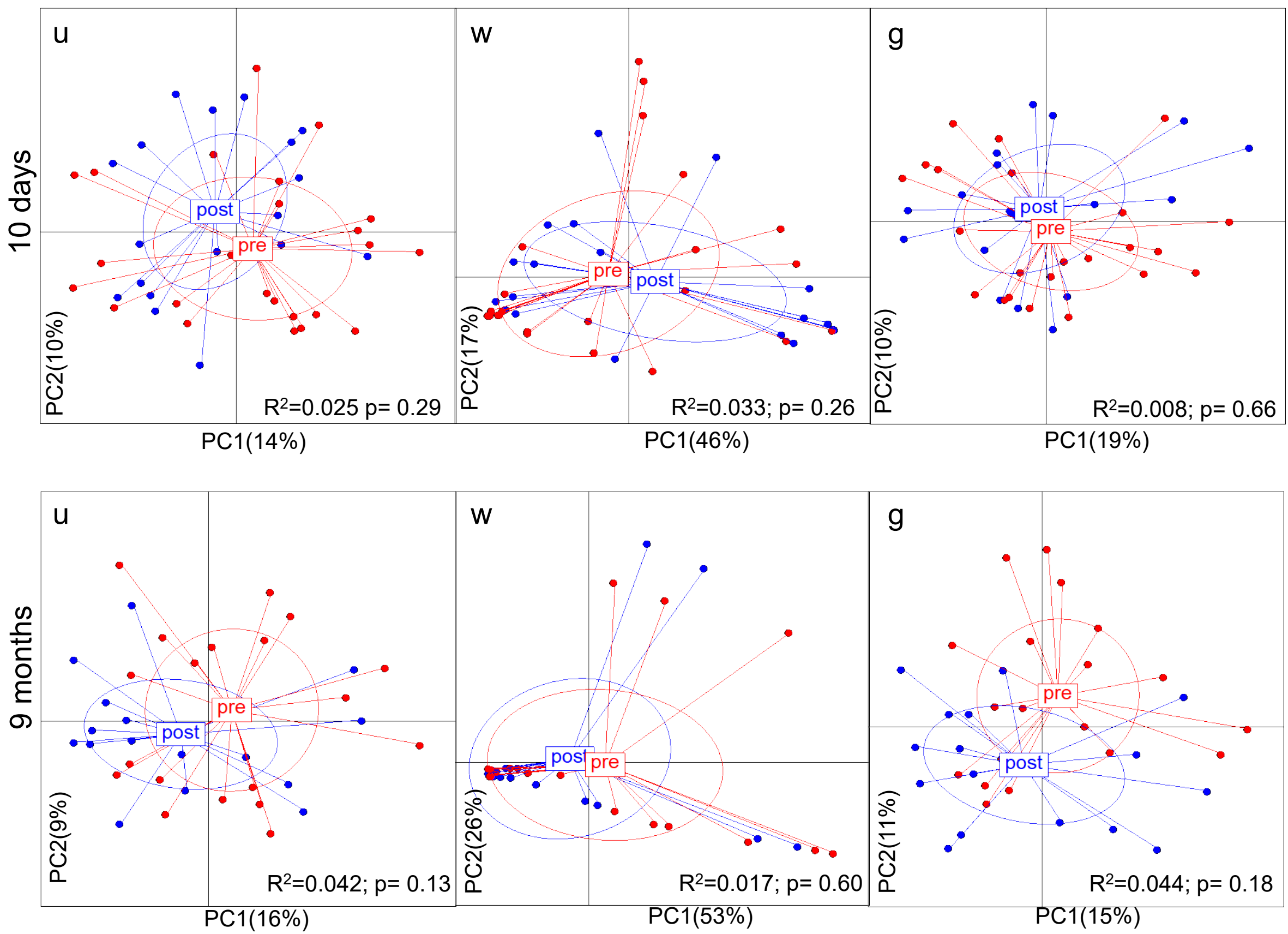
Figure. 4 (Online)
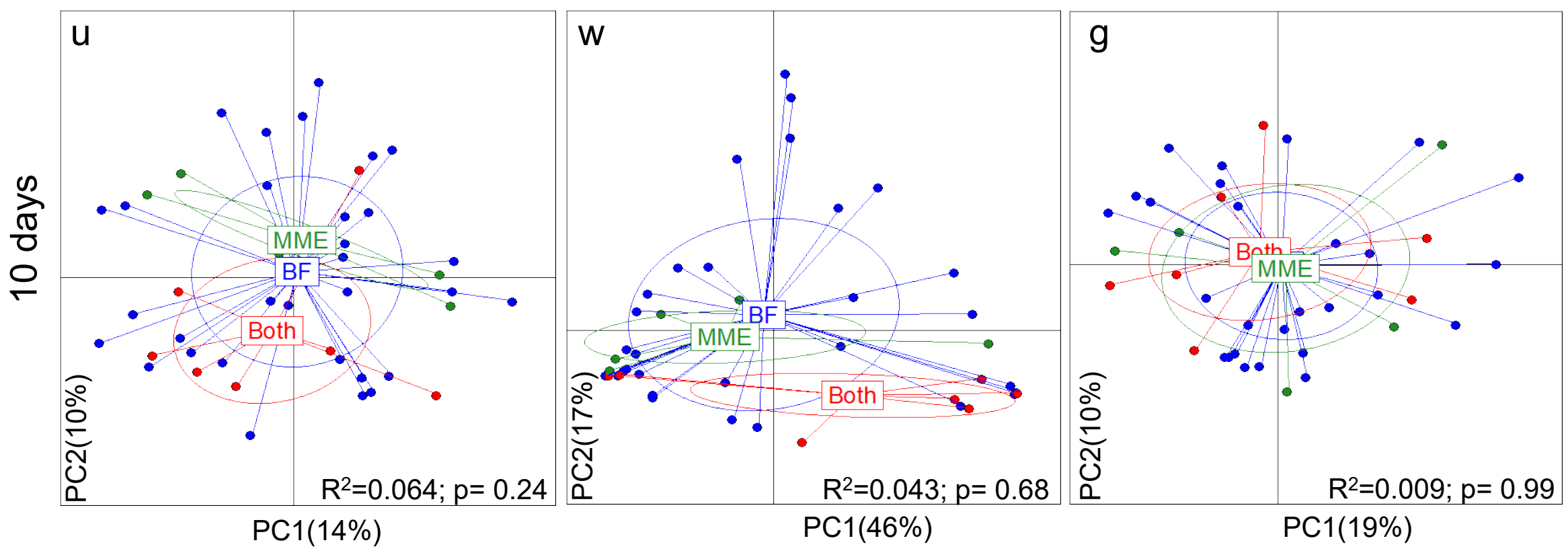


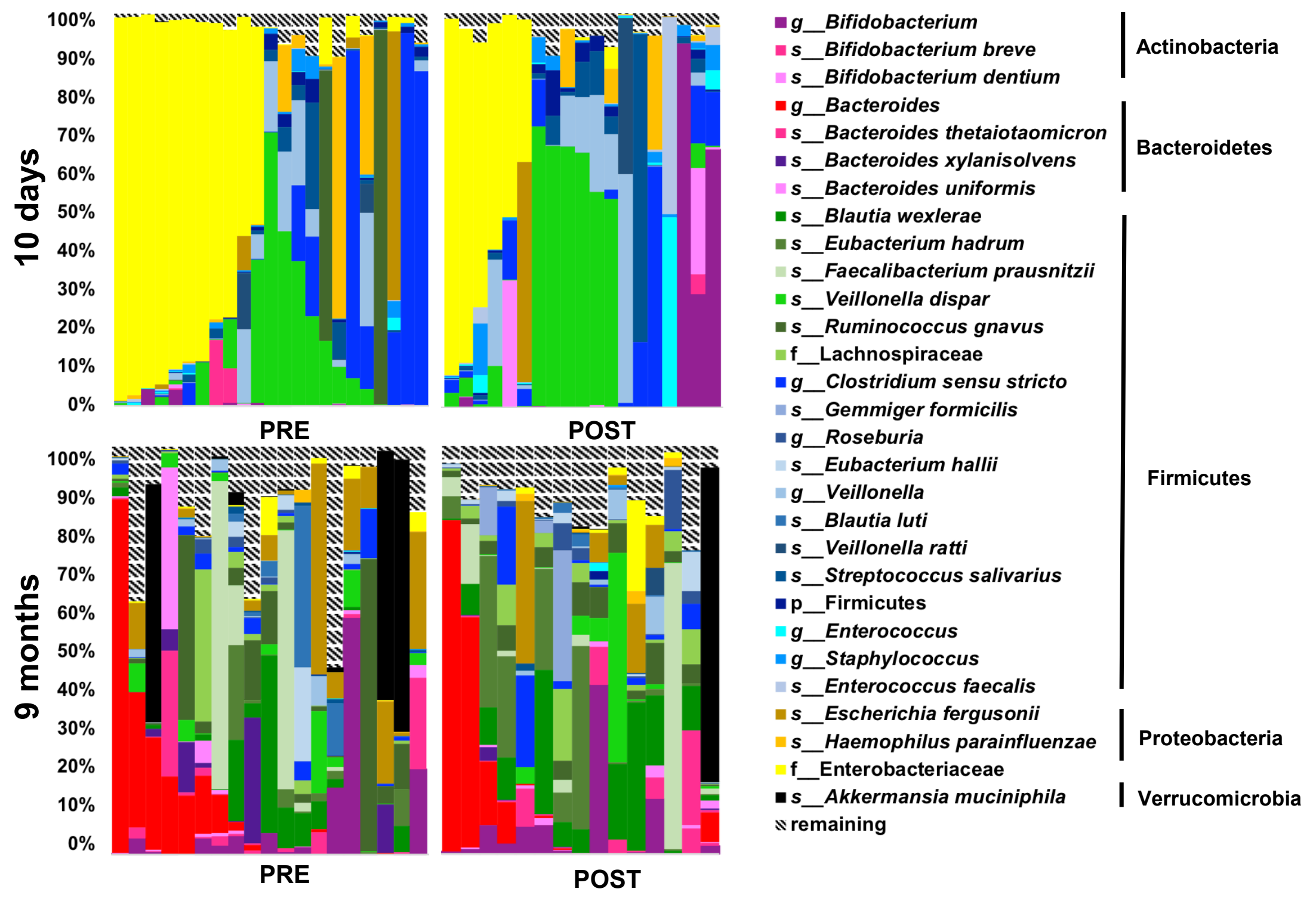

Figure 5 
Table 1 (Online). Distribution of bacterial isolated from fecal samples from 10 days old infants born by caesarean delivery for which mother was administered a single dose of cefuroxime either before (Pre) or after (Post) umbilical cord clamping. Isolates were identified by MALDI-TOF.

\begin{tabular}{lcc}
\hline \multirow{2}{*}{ Bacterial isolate } & \multicolumn{2}{c}{ Number tested (\%) } \\
\cline { 2 - 3 } & Pre & Post \\
\hline Gram positive bacteria & $\mathrm{N}=54$ & $\mathrm{~N}=31$ \\
Enterococcus spp. & 27 & 16 \\
Enterococcus faecalis & $20(21.3)$ & $16(32.7)$ \\
Other Enterococcus spp. ${ }^{*}$ & $7(7.4)$ & $0(0.0)$ \\
Staphylococcus spp. & 27 & 13 \\
$\quad$ Staphylococcus epidermidis & $16(17.0)$ & $8(16.3)$ \\
$\quad$ Staphylococcus aureus & $9(9.6)$ & $2(4.1)$ \\
Other Staphylococcus spp. ${ }^{* *}$ & $2(2.1)$ & $3(6.1)$ \\
Other gram-positive bacteria*** & $0(0.0)$ & $2(4.1)$ \\
Gram negative bacteria & & \\
Enterobacteriaceae & $\mathrm{N}=40$ & $\mathrm{~N}=18$ \\
$\quad$ Klebsiella pneumoniae & 39 & 17 \\
Enterobacter cloacae & $9(9.6)$ & $6(12.2)$ \\
Other Enterobacteriaceae† & $16(17.0)$ & $8(16.3)$ \\
Other gram-negative bacteria $\S$ & $14(14.9)$ & $3(6.1)$ \\
Total N=153 & $1(1.1)$ & $1(2.0)$ \\
\hline
\end{tabular}

* Enterococcus avium, Enterococcus maldoratus, Enterococcus faecium

** Staphylococcus hominis, Staphylococcus lugdunensis

*** Aerococcus viridans, Micrococcus lutues

† Enterobacter aerogenes, Enterobacter asburie, Klebsiella oxytoca, Citrobacter braakii, Citrobacter fruendii, Escherichia coli, Leclercia adecarboxylata

$\S$ Acinetobacter junii, Stenotrophomonas maltophilia 
Table 2 (online): Antibiotic susceptibility of Gram-positive bacteria isolated from feces 10 days after birth from babies born by cesarean delivery for which the mother was administered a single dose of cefuroxime either before (Pre) or after (Post) umbilical cord clamping. R, Resistant, I,

Intermediate, S, Susceptible, as determined by the Kirkby-Bauer test. NA, not applicable.

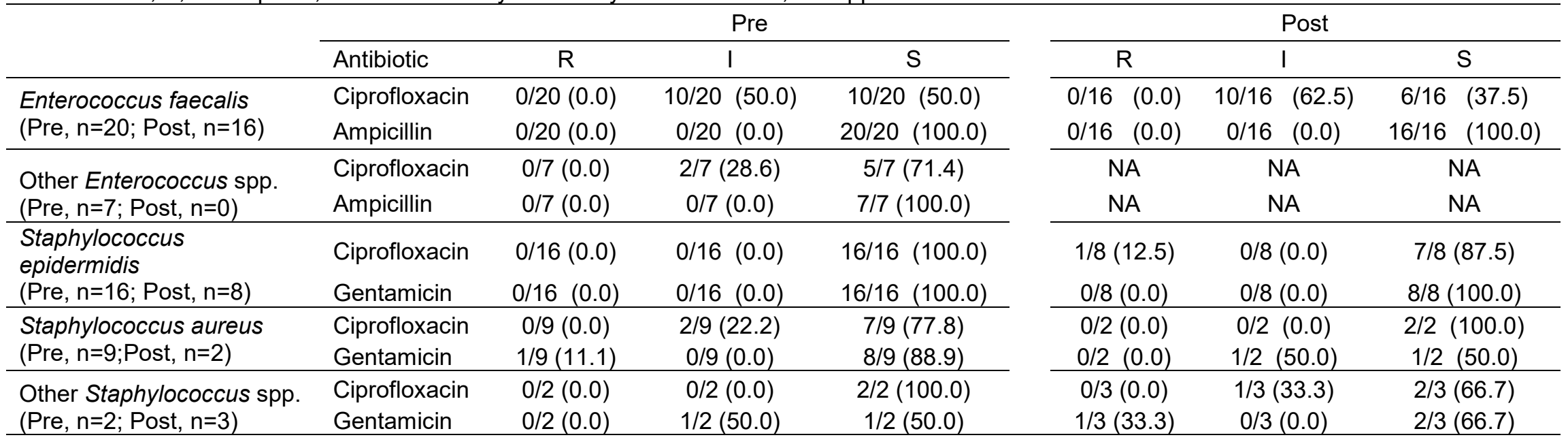


Table 3: Antibiotic susceptibility of gram-negative (Enterobacteriaceae) bacteria isolated from feces 10 days after birth from babies born by cesarean delivery for which the mother was administered a single dose of cefuroxime either before (Pre) or after (Post) umbilical cord clamping. R, Resistant, I, Intermediate, S, Susceptible, as determined by the Kirkby-Bauer test. NA, not applicable.

\begin{tabular}{|c|c|c|c|c|c|c|c|}
\hline & \multicolumn{4}{|c|}{ Pre } & \multicolumn{3}{|c|}{ Post } \\
\hline & Antibiotic & $\mathrm{R}$ & 1 & $S$ & $\mathrm{R}$ & I & S \\
\hline \multirow{5}{*}{$\begin{array}{l}\text { Klebsiella pneumoniae } \\
(\text { Pre, } n=9 ; \text { Post, } n=6)\end{array}$} & Cefuroxime & $0 / 9(0.0)$ & $1 / 9(11.1)$ & $8 / 9$ (88.9) & $0 / 6(0.0)$ & $0 / 6(0.0)$ & $6 / 6(100.0)$ \\
\hline & Ciprofloxacin & $0 / 9(0.0)$ & $1 / 9(11.1)$ & 8/9 (88.9) & $0 / 6(0.0)$ & $0 / 6(0.0)$ & $6 / 6(100.0)$ \\
\hline & Ampicillin & $5 / 9(55.6)$ & $3 / 9$ (33.3) & $1 / 9(11.1)$ & $3 / 6(50.0)$ & $3 / 6(50.0)$ & $0 / 6(0.0)$ \\
\hline & Gentamicin & $0 / 9(0.0)$ & $0 / 9(0.0)$ & 9/9 (100.0) & 0/6 (0.0) & $0 / 6(0.0)$ & $6 / 6(100.0)$ \\
\hline & $\begin{array}{l}\text { Pipercillin- } \\
\text { tazobactam }\end{array}$ & $0 / 9(0.0)$ & 1/9 (11.1) & 8/9 (88.9) & $0 / 6(0.0)$ & $0 / 6(0.0)$ & $6 / 6(100.0)$ \\
\hline \multirow{5}{*}{$\begin{array}{l}\text { Enterobacter cloacea } \\
(\text { Pre, } n=16 ; \text { Post, } n=8)\end{array}$} & Cefuroxime & $1 / 16(6.3)$ & $13 / 16(81.3)$ & $2 / 16(12.5)$ & $2 / 8(25.0)$ & $2 / 8(25.0)$ & $4 / 8(50.0)$ \\
\hline & Ciprofloxacin & $0 / 16(0.0)$ & $0 / 16(0.0)$ & $16 / 16(100.0)$ & $0 / 8(0.0)$ & $0 / 8(0.0)$ & $8 / 8(100.0)$ \\
\hline & Ampicillin & $7 / 16(43.8)$ & $4 / 16(25.0)$ & $5 / 16(31.3)$ & $5 / 8(62.5)$ & $2 / 8(25.0)$ & $1 / 8(12.5)$ \\
\hline & Gentamicin & $0 / 16(0.0)$ & $0 / 16(0.0)$ & $16 / 16(100.0)$ & $1 / 8(12.5)$ & $0 / 8(0.0)$ & $7 / 8(87.5)$ \\
\hline & $\begin{array}{l}\text { Pipercillin- } \\
\text { tazobactam }\end{array}$ & $1 / 16(6.3)$ & $2 / 16(12.5)$ & 13/16 (81.3) & $0 / 8(0.0)$ & $3 / 8(37.5)$ & $5 / 8(62.5)$ \\
\hline \multirow{5}{*}{$\begin{array}{l}\text { Other Enterobacteriaceae } \\
\text { (Pre, } n=14 ; \text { Post, } n=3 \text { ) }\end{array}$} & Cefuroxime & $0 / 14(0.0)$ & $0 / 14(0.0)$ & $14 / 14(100.0)$ & $0 / 3(0.0)$ & $0 / 3(0.0)$ & $3 / 3(100.0)$ \\
\hline & Ciprofloxacin & $0 / 14(0.0)$ & $0 / 14(0.0)$ & $14 / 14(100.0)$ & $0 / 3(0.0)$ & $0 / 3(0.0)$ & $3 / 3(100.0)$ \\
\hline & Ampicillin & $1 / 14(7.1)$ & $1 / 14(7.1)$ & $12 / 14(85.7)$ & $1 / 3(33.3)$ & $1 / 3(33.3)$ & $1 / 3(33.3)$ \\
\hline & Gentamicin & $0 / 14(0.0)$ & $0 / 14(0.0)$ & $14 / 14(100.0)$ & $0 / 3(0.0)$ & $0 / 3(0.0)$ & $3 / 3(100.0)$ \\
\hline & $\begin{array}{l}\text { Pipercillin- } \\
\text { tazobactam }\end{array}$ & $0 / 14(0.0)$ & $0 / 14(0.0)$ & $14 / 14(100.0)$ & $0 / 3(0.0)$ & $0 / 3(0.0)$ & $3 / 3(100.0)$ \\
\hline
\end{tabular}

